\title{
Design and Numerical Solutions of a Novel Third-Order Nonlinear Emden-Fowler Delay Differential Model
}

\author{
Juan L.G. Guirao $\mathbb{D}^{1},{ }^{1}$ Zulqurnain Sabir, ${ }^{2}$ and Tareq Saeed ${ }^{3}$ \\ ${ }^{1}$ Department of Applied Mathematics and Statistics, Technical University of Cartagena, Hospital de Santa Marina, \\ Cartagena 30203, Spain \\ ${ }^{2}$ Department of Mathematics and Statistics, Hazara University, Mansehra, Pakistan \\ ${ }^{3}$ Nonlinear Analysis and Applied Mathematics (NAAM)-Research Group, Department of Mathematics, Faculty of Science, \\ King Abdulaziz University, 80203, Jeddah 21589, Saudi Arabia
}

Correspondence should be addressed to Juan L.G. Guirao; juan.garcia@upct.es

Received 24 May 2020; Revised 14 August 2020; Accepted 17 August 2020; Published 27 August 2020

Academic Editor: Praveen Agarwal

Copyright (c) 2020 Juan L.G. Guirao et al. This is an open access article distributed under the Creative Commons Attribution License, which permits unrestricted use, distribution, and reproduction in any medium, provided the original work is properly cited.

\begin{abstract}
In this study, the design of a novel model based on nonlinear third-order Emden-Fowler delay differential (EF-DD) equations is presented along with two types using the sense of delay differential and standard form of the second-order EF equation. The singularity at $\xi=0$ at single or multiple points of each type of the designed EF-DD model are discussed. The detail of shape factors and delayed points is provided for both types of the designed third-order EF-DD model. For the verification and validation of the model, two numerical examples are presented of each case and numerical results have been performed using the artificial neural network along with the hybrid of global and local capabilities. The comparison of the obtained numerical results with the exact solutions shows the perfection and correctness of the designed third-order EF-DD model.
\end{abstract}

\section{Introduction}

The delay differential (DD) equation is known as one of the historical and important equations. Recently, DD equation has attained much attention of the researcher's community due to its vast applications in many biological models, as well as scientific phenomena such as communication system model, dynamical population model, economical systems, engineering system, and transport and propagation model [1-5]. It is always interested to find the solution of DD equations and many researchers have applied different numerical/analytical techniques. Brunner et al. [6] solved DD equation by applying a discontinuous Galerkin numerical scheme. Hsiao and $\mathrm{Wu}$ [7] applied Haar wavelet to solve DD equations, while Wang [8] presented the solution of DD equations using Legendre wavelet. Adomian and Rach [9] solved DD equation using the Adomian decomposition scheme. Shakeri and Dehghan [10] found the solutions of DD initial value problems using the homotopy perturbation scheme. Erdogan et al. [11] implemented finite difference approach on layer-adapted mesh using the singularly perturbed DD equations. The general form of the DD model is written as $[12,13]$

$$
\left\{\begin{array}{l}
\frac{\mathrm{d}^{3} u}{\mathrm{~d} \xi^{3}}=h\left(\xi, u(\xi-\tau), \frac{\mathrm{d} u(\xi-\tau)}{\mathrm{d} \xi}, \frac{\mathrm{d}^{2} u(\xi-\tau)}{\mathrm{d} \xi^{2}}\right), \\
u(0)=A, \frac{\mathrm{d} u(0)}{\mathrm{d} \xi}=B, \frac{\mathrm{d}^{2} u(0)}{\mathrm{d} \xi^{2}}=C,
\end{array}\right.
$$

where $h$ shows the linear/nonlinear function and $\tau$ is the delayed term, whereas $A, B$, and $C$ are the constants.

The singular study has become very significant in the modern era due to the variety of applications in technology, engineering, and biological and physical sciences. The singular nature models are always difficult, grim, and challengeable to solve for the research community. One of the important, famous, historical, and singular models is 
Emden-Fowler (EF) model that shows the singularity at the origin. Since its invention, this model has been solved by various analytical and numerical schemes, and it has a number of applications in the study of relativistic mechanics, fluid dynamics, population growth model, pattern creation, and the study of chemical reactor models. The literature form of the EF model is written as [14-16]

$$
\left\{\begin{array}{l}
\frac{\mathrm{d}^{2} u}{\mathrm{~d} \xi^{2}}+\frac{\kappa}{\xi} \frac{\mathrm{d} u}{\mathrm{~d} \xi}+g(\xi) h(u)=0, \\
u(0)=A_{1}, \frac{\mathrm{d} u(0)}{\mathrm{d} \xi}=A_{2},
\end{array}\right.
$$

where $\kappa \geq 1$ is the shape vector. The EF model (1) becomes the Lane-Emden model by taking $h(u)=1$ and is written as follows:

$$
\left\{\begin{array}{l}
\frac{\mathrm{d}^{2} u}{\mathrm{~d} \xi^{2}}+\frac{\kappa}{\xi} \frac{\mathrm{d} u}{\mathrm{~d} \xi}+h(u)=0 \\
u(0)=A_{1}, \frac{\mathrm{d} u(0)}{\mathrm{d} \xi}=A_{2}
\end{array}\right.
$$

The above singular models have been achieved from the work of Homer Lane and Robert Emden. These models designate inner construction of polytropic stars, gas cloud model, cluster galaxies, and radiative cooling. Due to the worth of these models, no one can deny the value and importance of such models, which has vast applications in the physical science field [17], isotropic continuous media [18], density of gaseous star [19], morphogenesis [20], dusty fluid models [21], stellar structure models [22], reactions based on catalytic diffusion [23], oscillating magnetic systems [24], isothermal gas sphere models [25], mathematical physics [26], catalytic diffusion reactions [23], classical/ quantum mechanics [27], and electromagnetic theory [28].

Due to the fame of these models, the researcher's community is interested to solve these models and only a few methods are available in the literature that has been investigated. One of the well-known methods used to solve these models is the Adomian decomposition method, which is proposed by Shawagfeh and Wazwaz $[29,30]$. Parand and Razzaghi [31] implemented a famous numerical scheme to solve singular equations. Liao [32] applied an analytic technique to avoid the difficulty of singular points. Bender et al. [33] proposed a perturbative scheme to solve the singular models. Nouh [34] presented two techniques' power series and Pade approximation to solve the singular models.

The aim of this study is to design a novel third-order Emden-Fowler delay differential (EF-DD) model along with two types. Two examples of the designed third-order EF-DD model have been presented for both of the types. For the correctness of the model, the numerical investigations have been performed by using an artificial neural network along with its global/local competences. The singular ordinary differential equations are much important and have many applications in engineering as well as scientific applications, e.g., optimization and control theory, reactant application in the area of chemical reactor, theory of boundary layer, and biological sciences.

The structure of remaining paper is summarized as follows. Section 2 defines the construction of the third-order EF-DD model along with two types. Methodology and the detail of the results for solving the third-order EF-DD equations are provided in the Section 3. The conclusions along with future research directions are drawn in the Section 4.

\section{Construction of Third-Order EF-DD Model}

In this section, two different types are presented based on the third-order EF-DD model. The construction of the thirdorder EF-DD model along with the singular points, delayed points, and shape factors for both of the types is discussed. The initial conditions of the designed third-order EF-DD model are achieved using the standard form of the Lane-Emden. To derive the third-order EF-DD model system of Emden-Fowler equations, the mathematical form is used as follows:

$$
\xi^{-k} \frac{\mathrm{d}^{p}}{\mathrm{~d} \xi^{p}}\left(\xi^{k} \frac{\mathrm{d}^{q}}{\mathrm{~d} \xi^{q}}\right) u(\xi-\tau)+g(\xi) h(u)=0,
$$

where $k$ is real positive number. To determine the thirdorder DD-EF model, the values of $p$ and $q$ should be designated as follows:

$$
p+q=3, \quad p, q \geq 1 .
$$

The following two possibilities satisfy equation (5) as follows:

$$
\begin{aligned}
& p=2, \\
& q=1, \\
& p=1, \\
& q=2 .
\end{aligned}
$$

2.1. Type 1. Using equations (6), the updated form of equation (4) is

$$
\xi^{-k} \frac{\mathrm{d}^{2}}{\mathrm{~d} \xi^{2}}\left(\xi^{k} \frac{\mathrm{d}}{\mathrm{d} \xi}\right) y(\xi-\tau)+g(\xi) h(u)=0 .
$$

The derivative part of the above equation is obtained as follows:

$$
\begin{aligned}
& \frac{\mathrm{d}^{2}}{\mathrm{~d} \xi^{2}}\left(\xi^{k} \frac{\mathrm{d}}{\mathrm{d} \xi}\right) u(\xi-\tau)=\xi^{k} \frac{\mathrm{d}^{3}}{\mathrm{~d} \xi^{3}} u(\xi-\tau)+2 k \xi^{k-1} \\
& \frac{\mathrm{d}^{2}}{\mathrm{~d} \xi^{2}} u(\xi-\tau)+k(k-1) u(\xi-\tau) \xi^{k-2} \frac{\mathrm{d}}{\mathrm{d} \xi} u(\xi-\tau)
\end{aligned}
$$

Using the above expression in equation (8), the thirdorder EF-DD equation becomes 


$$
\left\{\begin{array}{l}
\frac{\mathrm{d}^{3}}{\mathrm{~d} \xi^{3}} u(\xi-\tau)+\frac{2 k}{\xi} \frac{\mathrm{d}^{2}}{\mathrm{~d} \xi^{2}} u(\xi-\tau)+\frac{k(k-1)}{\xi^{2}} \frac{\mathrm{d}}{\mathrm{d} \xi} u(\xi-\tau)+g(\xi) h(u)=0, \\
u(0)=\alpha, \frac{\mathrm{d} u(0)}{\mathrm{d} \xi}=0, \frac{\mathrm{d}^{2} u(0)}{\mathrm{d} \xi^{2}}=0,
\end{array}\right.
$$

where the singular point at $\xi=0$ appears two times as $\xi=0$ and $\xi^{2}=0$. The shape factors expressed in equation (10) are $2 k$ and $k(k-1)$, respectively. The multiple delays have been noticed in the first, second, and third term of equation (10). Moreover, the third expression vanishes for $k=1$ and the shape factor reduces to 2 .

2.2. Type 2. Equation (4) by putting $p=1$ and $q=2$ takes the form as follows:

$$
\xi^{-k} \frac{\mathrm{d}}{\mathrm{d} \xi}\left(\xi^{k} \frac{\mathrm{d}^{2}}{\mathrm{~d} \xi^{2}}\right) u(\xi-\tau)+g(\xi) h(u)=0 .
$$
follows:

The derivative part of the above equation is obtained as

$$
\frac{\mathrm{d}}{\mathrm{d} \xi}\left(\xi^{k} \frac{\mathrm{d}^{2}}{\mathrm{~d} \xi^{2}}\right) u(\xi-\tau)=\xi^{k} \frac{\mathrm{d}^{3}}{\mathrm{~d} \xi^{3}} u(\xi-\tau)+k \xi^{k-1} \frac{\mathrm{d}^{2}}{\mathrm{~d} \xi^{2}} u(\xi-\tau) .
$$

Using the above value in equation (11), the third-order EF-DD model becomes as follows:

$$
\left\{\begin{array}{l}
\frac{\mathrm{d}^{3}}{\mathrm{~d} \xi^{3}} u(\xi-\tau)+\frac{k}{\xi} \frac{\mathrm{d}^{2}}{\mathrm{~d} \xi^{2}} u(\xi-\tau)+g(\xi) h(u)=0, \\
u(0)=\alpha, \frac{\mathrm{d} u(0)}{\mathrm{d} \xi}=\beta, \frac{\mathrm{d}^{2} u(0)}{\mathrm{d} \xi^{2}}=0 .
\end{array}\right.
$$

The single singularity at $\xi=0$ has been noticed in the above equation (13). The shape factor is $k$ and delayed expression appears twice in the above equation.

Some prime features of the designed model are presented as follows:

The design of third-order Emden-Fowler delay differential model is presented by using the sense of standard Emden-Fowler equation and delay-differential equation

Two types of the designed model are presented and two numerical nonlinear examples of each type are designed based on the designed model

The shape factors, delay expressions, and singularities are discussed in both of the types

The artificial neural network is used to check the perfection and correctness of the designed third-order Emden-Fowler model

\section{Methodology and Numerical Examples}

Two numerical examples based on the EF-DD novel model are presented in this section. The numerical investigations of the examples are performed using the artificial neural network. The error function is provided by using the sense of the differential equations and initial conditions. The optimization of the error function is performed using the hybrid of global and local search captaincies, which are genetic algorithm (GA) and active-set method (ASM). The artificial neural network is famous and widely applied in many wellknown recent applications, see [35-41]. To approximate the results, feedforward ANN system along with its respective derivatives is used as follows:

$$
\begin{gathered}
\widehat{u}=\sum_{i=1}^{m} l_{i} P\left(\alpha_{i} \xi+b_{i}\right), \\
\widehat{u}^{(n)}=\sum_{i=1}^{m} l_{i} P^{(n)}\left(\alpha_{i} \xi+b_{i}\right),
\end{gathered}
$$

where $l_{i}, m_{i}$, and $n_{i}$ are the $i$ th components of $l, \alpha$, and $b$ vectors, while $n$ is the order of derivative. An activation logsigmoid function, i.e., $P(\xi)=\left(1+\& \text { ExponentialE } ;^{-\xi}\right)^{-1}$ along with its third derivative is used as follows:

$$
\begin{aligned}
\widehat{u} & =\sum_{i=1}^{m} l_{i}\left(1+e^{-\left(\alpha_{i} \xi+b_{i}\right)}\right)^{-1}, \\
\widehat{u}^{(n)} & =\sum_{i=1}^{n} l_{i} \frac{\mathrm{d}^{n}}{\mathrm{~d} \xi^{n}}\left(\left(1+e^{-\left(\alpha_{i} \xi+b_{i}\right)}\right)^{-1}\right) .
\end{aligned}
$$

The third-order derivative is provided as follows:

$$
\begin{aligned}
\widehat{u}^{\prime \prime \prime}(\xi)= & \sum_{i=1}^{m} l_{i} \xi_{i}^{3}\left(\frac{6 e^{-3\left(\alpha_{i} \xi+b_{i}\right)}}{\left(1+e^{-\left(\alpha_{i} \xi+b_{i}\right)}\right)^{4}}-\frac{6 e^{-2\left(\alpha_{i} \xi+b_{i}\right)}}{\left(1+e^{-\left(\alpha_{i} \xi+b_{i}\right)}\right)^{3}}\right. \\
& \left.+\frac{e^{-\left(\alpha_{i} \xi+b_{i}\right)}}{\left(1+e^{-\left(\alpha_{i} \xi+b_{i}\right)}\right)^{2}}\right) .
\end{aligned}
$$

The fitness function is given as follows:

$$
E=E_{1}+E_{2}
$$

where $E_{1}$ and $E_{2}$ are the respective error functions related to differential equation and initial conditions. 

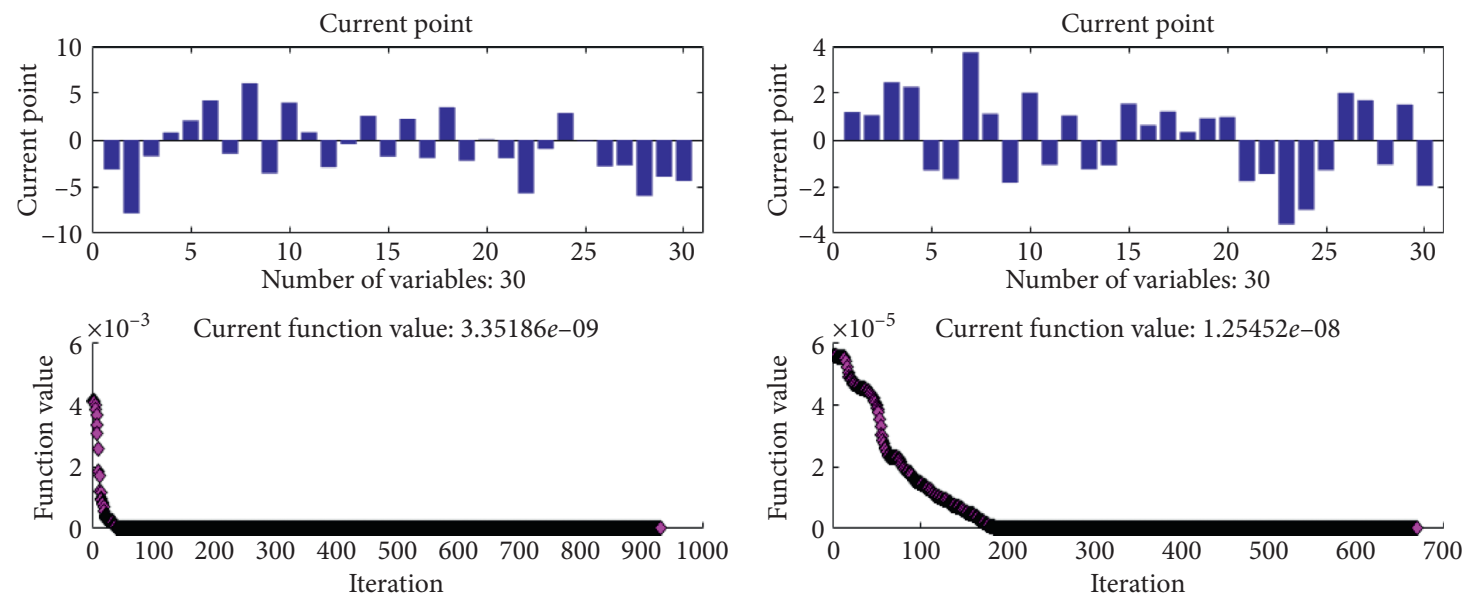

(a)

(b)

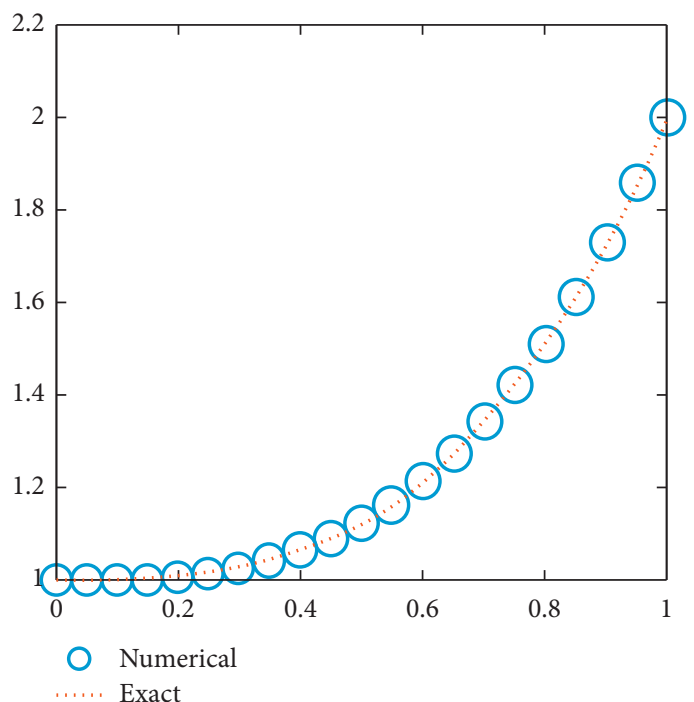

(c)

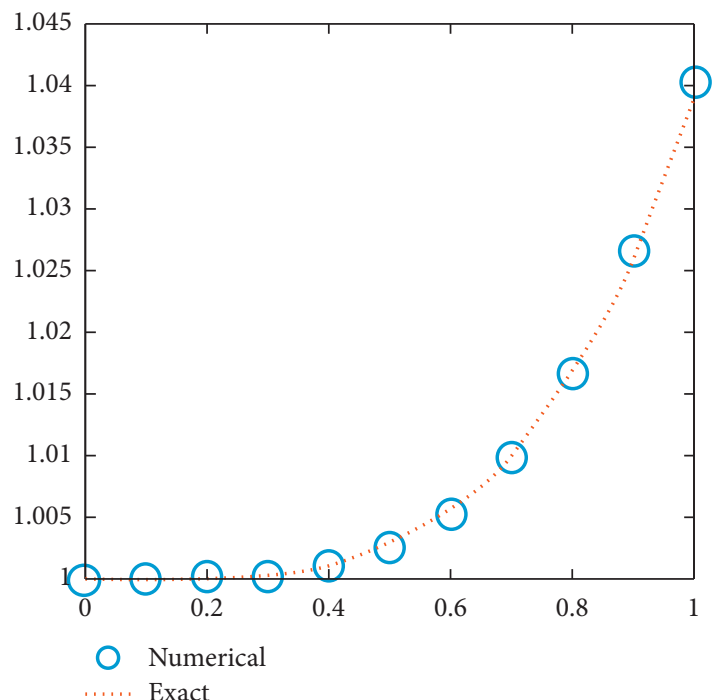

(d)

FIGURE 1: Optimization variables, learning curves, and comparison of results of the GA-AS scheme for nonlinear EF-DD equations (1) and (2) of type 1. (a) Set of best weights and current function values for 10 neurons based on third-order nonlinear EF-DD equation (1). (b) Set of best weights and current function values for 10 neurons based on third-order nonlinear EF-DD equation (2). (c) Comparison of the numerical and exact solutions of third-order nonlinear EF-DD equation (1). (d) Comparison of the numerical and exact solutions of thirdorder nonlinear EF-DD equation (2).

3.1. EF-DD Equation of Type 1. In this type, two different third-order EF-DD-based equations will be discussed. The updated form of equation (10) using $k=2$ is given as follows.
Example 1. Consider the nonlinear third-order EF-DD equation having multiple singularities is shown as follows:

$$
\left\{\begin{array}{l}
\frac{\mathrm{d}^{3}}{\mathrm{~d} \xi^{3}} u(\xi-1)+\frac{4}{\xi} \frac{\mathrm{d}^{2}}{\mathrm{~d} \xi^{2}} u(\xi-1)+\frac{2}{\xi^{2}} \frac{\mathrm{d}}{\mathrm{d} \xi} u(\xi-1)+\xi u^{2}=\xi^{7}+2 \xi^{4}+\xi+30-\frac{36}{\xi}+\frac{6}{\xi^{2}} \\
u(0)=1, \frac{\mathrm{d} u(0)}{\mathrm{d} \xi}=0, \frac{\mathrm{d}^{2} u(0)}{\mathrm{d} \xi^{2}}=0 .
\end{array}\right.
$$



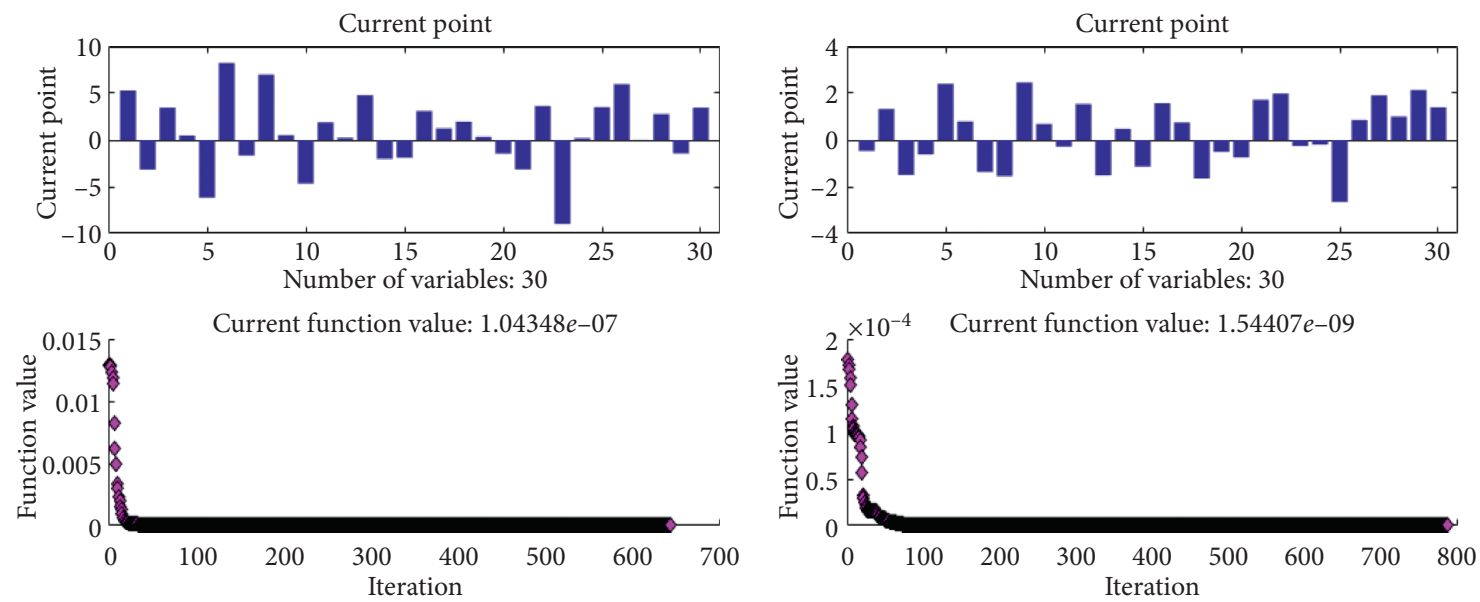

(a)

(b)

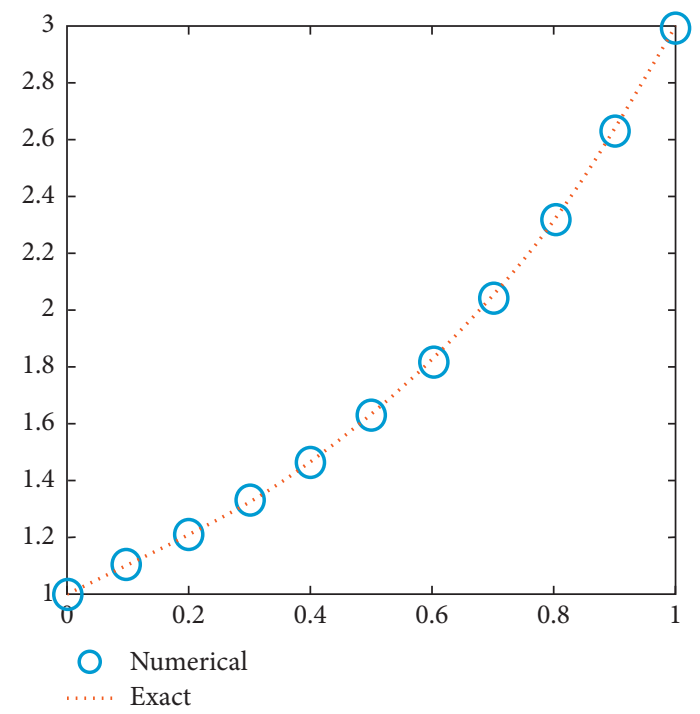

(c)

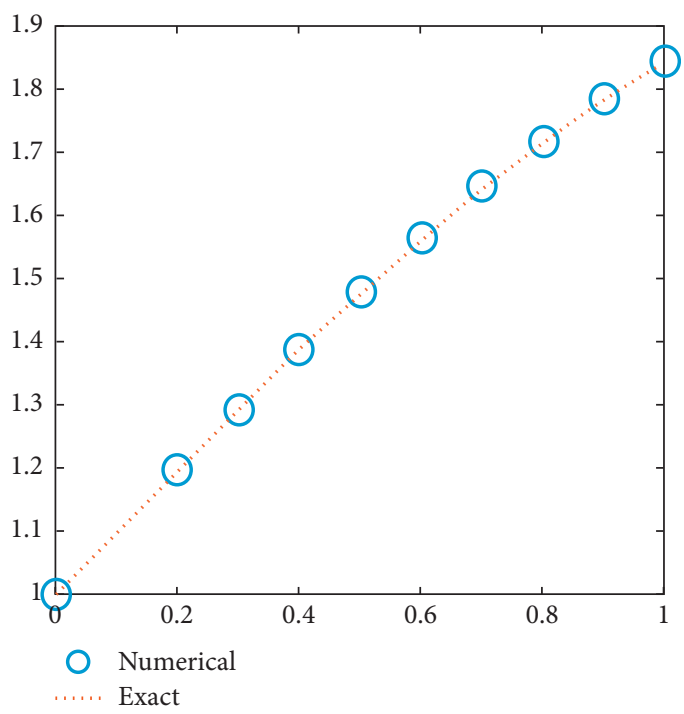

(d)

FIgURE 2: Optimization variables, learning curves, and comparison of results of the GA-AS scheme for nonlinear EF-DD equations (1) and (2) of type 2. (a) Set of best weights and current function values for 10 neurons based on third-order nonlinear EF-DD equation (1) of type 2. (b) Set of best weights and current function values for 10 neurons based on third-order nonlinear EF-DD equation (2) of type 2. (c) Comparison of the numerical and exact solutions of third-order nonlinear EF-DD equation (1) of type 2. (d) Comparison of the numerical and exact solutions of third-order nonlinear EF-DD equation (2) of type 2.

The exact solution of equation (20) is $1+\xi^{3}$.

Example 2. Consider the nonlinear third-order EF-DD equation having multiple singularities and trigonometric functions is written as follows:

$$
\left\{\begin{array}{l}
\frac{\mathrm{d}^{3}}{\mathrm{~d} \xi^{3}} u(\xi-1)+\frac{4}{\xi} \frac{\mathrm{d}^{2}}{\mathrm{~d} \xi^{2}} u(\xi-1)+\frac{2}{\xi^{2}} \frac{\mathrm{d}}{\mathrm{d} \xi} u(\xi-1)+\xi u^{2}=\frac{\xi^{5}}{4}-\frac{2}{\xi^{2}}+\frac{6}{\xi}+ \\
\frac{\xi^{2}-2}{\xi^{2}} \sin (\xi-1)-\frac{4}{\xi} \cos (\xi-1)+\xi^{3} \cos \xi+\xi \cos ^{2} \xi, \\
u(0)=1, \frac{\mathrm{d} u(0)}{\mathrm{d} \xi}=0, \frac{\mathrm{d}^{2} u(0)}{\mathrm{d} \xi^{2}}=0 .
\end{array}\right.
$$

The exact solution of equation $(21)$ is $\cos \xi+(1 / 2) \xi^{2}$.

3.2. EF-DD Equation of Type 2. In this type, two different third-order EF-DD-based equations will be discussed. The updated form of equation (13) using $k=1$ is given in the form of two examples.

Example 3. Consider the nonlinear third-order EF-DD equation having exponential function is given as follows:

$$
\left\{\begin{array}{l}
\frac{\mathrm{d}^{3}}{\mathrm{~d} \xi^{3}} u(\xi-1)+\frac{1}{\xi} \frac{\mathrm{d}^{2}}{\mathrm{~d} \xi^{2}} u(\xi-1)+\xi e^{u}=12-\frac{6}{\xi}+\xi e^{1+\xi+\xi^{3}} \\
u(0)=1, \frac{\mathrm{d} u(0)}{\mathrm{d} \xi}=1, \frac{\mathrm{d}^{2} u(0)}{\mathrm{d} \xi^{2}}=0 .
\end{array}\right.
$$




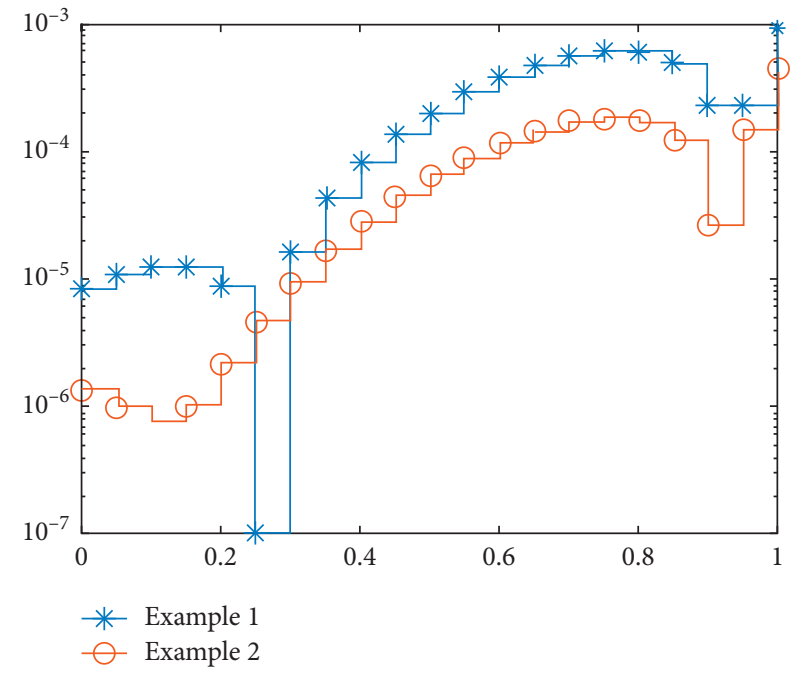

(a)

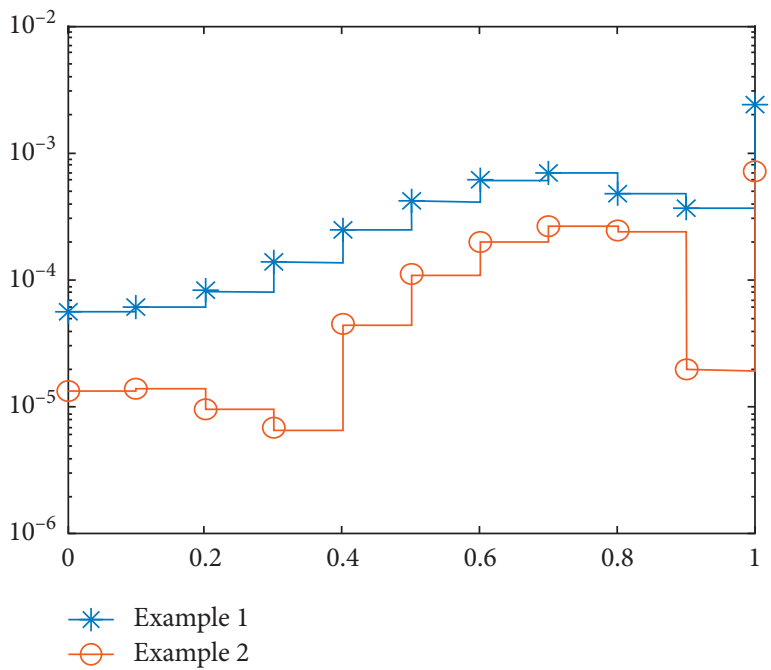

(b)

Figure 3: Absolute error based on the nonlinear EF-DD equations (1) and (2) of types 1 and 2. (a) AE for examples 1 and 2 of type 1. (b) AE for examples 1 and 2 of type 2 .

The exact solution of equation (22) is $1+\xi+\xi^{3}$.
Example 4. Consider the nonlinear third-order EF-DD equation having multi trigonometric function is given as follows:

$$
\left\{\begin{array}{l}
\frac{\mathrm{d}^{3}}{\mathrm{~d} \xi^{3}} u(\xi-1)+\frac{1}{\xi} \frac{\mathrm{d}^{2}}{\mathrm{~d} \xi^{2}} u(\xi-1)+\xi u^{2}=\xi \sin ^{2} \xi+2 \xi \sin \xi+\xi-\cos (\xi-1)-\frac{1}{\xi} \sin (\xi-1), \\
u(0)=1, \frac{\mathrm{d} u(0)}{\mathrm{d} \xi}=1, \frac{\mathrm{d}^{2} u(0)}{\mathrm{d} \xi^{2}}=0 .
\end{array}\right.
$$

The exact solution of equation (23) is $1+\sin \xi$.

Figures 1 and 2 represent the current point and function values using 10 neurons based on the hybrid combination of GA-AS scheme for both of the examples of types 1 and 2. The current function values (CFVs) are $10^{-09}$ and $10^{-08}$ for both of the examples of type 1 and $10^{-07}$ and $10^{-09}$ for both of the examples of 2 using 10 numbers of neurons. The comparison of results is presented in the rest of the figures for both examples of types 1 and 2. The overlapping of the exact and obtained results shows the correctness and the perfection of the novel third-order nonlinear EF-DD model.

The plots of the absolute error (AE) for both types of examples 1 and 2 based on the third-order nonlinear EF-DD model are provided in Figure 3. It is clear that most of the values lie around $10^{-04}$ to $10^{-05}$ for both types of examples 1 and 2, which indicates the exactness of the designed model. These witnesses prove the correctness of the designed thirdorder nonlinear EF-DD model. Comparison of the obtained results from GA-ASM for solving the nonlinear EF-DD model based on both problems of both types is tabulated in
Tables 1 and 2. The exact solution, proposed results from GA-ASM, and the AE are provided in these tables. One can conclude on the behalf of AE the exactness and accurateness of the proposed model, as well as designed scheme.

\section{Conclusion}

In the present study, a novel design of third-order Emden-Fowler delay differential model is presented. The designed model is obtained by using the sense of fundamental Emden-Fowler model. The details of singular points, delay expressions, and the shape factors are also provided of the modeled equations of each type. The singularity at $\xi=0$ appears twice in the first type, while single singularity is noticed in the second type. Similarly, the shape factor is unique in the standard form of the Emden-Fowler model, while the occurrence of shape factor is noticed twice in the type 1; however, single shape factor is noticed in type 2. For the perfection of the designed model, two nonlinear examples are presented of each type and numerical 
TABLE 1: Comparison of the obtained results from GA-ASM for solving the nonlinear EF-DD model based on both problems of type 1.

\begin{tabular}{|c|c|c|c|c|c|c|}
\hline \multirow{2}{*}{$\xi$} & \multicolumn{3}{|c|}{ Problem I } & \multicolumn{3}{|c|}{ Problem 2} \\
\hline & Exact & GA-ASM & $\mathrm{AE}$ & Exact & GA-ASM & $\mathrm{AE}$ \\
\hline 0 & 1.00000000 & 0.99998788 & $1.212101 E-05$ & 1.00000000 & 0.99999570 & $4.2979068 E-06$ \\
\hline 0.05 & 1.00012500 & 1.00010387 & $2.112830 E-05$ & 1.00000026 & 0.99999586 & $4.4025337 E-06$ \\
\hline 0.1 & 1.00100000 & 1.00097307 & $2.693406 E-05$ & 1.00000417 & 0.99999959 & $4.5773499 E-06$ \\
\hline 0.15 & 1.00337500 & 1.00335045 & $2.454778 E-05$ & 1.00002108 & 1.00001622 & $4.8547562 E-06$ \\
\hline 0.2 & 1.00800000 & 1.00799333 & $6.670848 E-06$ & 1.00006658 & 1.00006131 & $5.2678390 E-06$ \\
\hline 0.25 & 1.01562500 & 1.01566134 & $3.633505 E-05$ & 1.00016242 & 1.00015658 & $5.8417298 E-06$ \\
\hline 0.3 & 1.02700000 & 1.02711600 & $1.160012 E-04$ & 1.00033649 & 1.00032991 & $6.5812201 E-06$ \\
\hline 0.35 & 1.04287500 & 1.04311966 & $2.446627 E-04$ & 1.00062271 & 1.00061526 & $7.4548770 E-06$ \\
\hline 0.4 & 1.06400000 & 1.06443360 & $4.336027 E-04$ & 1.00106099 & 1.00105262 & $8.3764421 E-06$ \\
\hline 0.45 & 1.09112500 & 1.09181538 & $6.903829 E-04$ & 1.00169710 & 1.00168792 & $9.1849453 E-06$ \\
\hline 0.5 & 1.12500000 & 1.12601542 & $1.015419 E-03$ & 1.00258256 & 1.00257294 & $9.6257224 E-06$ \\
\hline 0.55 & 1.16637500 & 1.16777299 & $1.397990 E-03$ & 1.00377452 & 1.00376519 & $9.3353752 E-06$ \\
\hline 0.6 & 1.21600000 & 1.21781202 & $1.812016 E-03$ & 1.00533561 & 1.00532778 & $7.8346627 E-06$ \\
\hline 0.65 & 1.27462500 & 1.27683704 & $2.212044 E-03$ & 1.00733380 & 1.00732926 & $4.5343334 E-06$ \\
\hline 0.7 & 1.34300000 & 1.34552994 & $2.529940 E-03$ & 1.00984219 & 1.00984343 & $1.2400050 E-06$ \\
\hline 0.75 & 1.42187500 & 1.42454775 & $2.672747 E-03$ & 1.01293887 & 1.01294907 & $1.0196762 E-05$ \\
\hline 0.8 & 1.51200000 & 1.51452198 & $2.521980 E-03$ & 1.01670671 & 1.01672970 & $2.2992646 E-05$ \\
\hline 0.85 & 1.61412500 & 1.61605929 & $1.934293 E-03$ & 1.02123315 & 1.02127325 & $4.0102911 E-05$ \\
\hline 0.9 & 1.72900000 & 1.72974297 & $7.429666 E-04$ & 1.02660997 & 1.02667161 & $6.1637149 E-05$ \\
\hline 0.95 & 1.85737500 & 1.85613407 & $1.240925 E-03$ & 1.03293309 & 1.03302018 & $8.7089331 E-05$ \\
\hline 1 & 2.00000000 & 1.99577063 & $4.229373 E-03$ & 1.04030231 & 1.04041732 & $1.1501021 E-04$ \\
\hline
\end{tabular}

TABLE 2: Comparison of the obtained results from GA-ASM for solving the nonlinear EF-DD model based on both problems of type 2.

\begin{tabular}{|c|c|c|c|c|c|c|}
\hline \multirow{2}{*}{$\xi$} & \multicolumn{3}{|c|}{ Problem I } & \multicolumn{3}{|c|}{ Problem 2} \\
\hline & Exact & GA-ASM & $\mathrm{AE}$ & Exact & GA-ASM & $\mathrm{AE}$ \\
\hline 0 & 1.00000000 & 1.00022916 & $2.291613 E-04$ & 1.00000000 & 0.99963919 & $3.60806 E-04$ \\
\hline 0.05 & 1.05012500 & 1.05035972 & $2.347211 E-04$ & 1.04997917 & 1.04960463 & $3.74535 E-04$ \\
\hline 0.1 & 1.10100000 & 1.10123006 & $2.300602 E-04$ & 1.09983342 & 1.09944214 & $3.91281 E-04$ \\
\hline 0.15 & 1.15337500 & 1.15357541 & $2.004064 E-04$ & 1.14943813 & 1.14902494 & $4.13190 E-04$ \\
\hline 0.2 & 1.20800000 & 1.20812735 & $1.273485 E-4$ & 1.19866933 & 1.19822683 & $4.42503 E-04$ \\
\hline 0.25 & 1.26562500 & 1.26561619 & $8.813729 E-06$ & 1.24740396 & 1.24692273 & $4.81224 E-04$ \\
\hline 0.3 & 1.32700000 & 1.32677477 & $2.252260 E-04$ & 1.29552021 & 1.29498952 & $5.30689 E-04$ \\
\hline 0.35 & 1.39287500 & 1.39234339 & $5.316061 E-04$ & 1.34289781 & 1.34230679 & $5.91015 E-04$ \\
\hline 0.4 & 1.46400000 & 1.46307475 & $9.252507 E-04$ & 1.38941834 & 1.38875787 & $6.60468 E-04$ \\
\hline 0.45 & 1.54112500 & 1.53973795 & $1.387048 E-03$ & 1.43496553 & 1.43423077 & $7.34760 E-04$ \\
\hline 0.5 & 1.62500000 & 1.62312048 & $1.879521 E-03$ & 1.47942554 & 1.47861923 & $8.06304 E-04$ \\
\hline 0.55 & 1.71637500 & 1.71402744 & $2.347561 E-03$ & 1.52268723 & 1.52182376 & $8.63464 E-04$ \\
\hline 0.6 & 1.81600000 & 1.81327792 & $2.722078 E-03$ & 1.56464247 & 1.56375263 & $8.89845 E-04$ \\
\hline 0.65 & 1.92462500 & 1.92169852 & $2.926483 E-03$ & 1.60518641 & 1.60432276 & $8.63645 E-04$ \\
\hline 0.7 & 2.04300000 & 2.04011427 & $2.885735 E-03$ & 1.64421769 & 1.64346056 & $7.57124 E-04$ \\
\hline 0.75 & 2.17187500 & 2.16933723 & $2.537774 E-03$ & 1.68163876 & 1.68110255 & $5.36205 E-04$ \\
\hline 0.8 & 2.31200000 & 2.31015280 & $1.847204 E-03$ & 1.71735609 & 1.71719584 & $1.60246 E-04$ \\
\hline 0.85 & 2.46412500 & 2.46330377 & $8.212322 E-04$ & 1.75128041 & 1.75169842 & $4.18013 E-04$ \\
\hline 0.9 & 2.62900000 & 2.62947213 & $4.721265 E-04$ & 1.78332691 & 1.78457923 & $1.25232 E-03$ \\
\hline 0.95 & 2.80737500 & 2.80925864 & $1.883638 E-03$ & 1.81341550 & 1.81581807 & $2.40257 E-03$ \\
\hline 1 & 3.00000000 & 3.00316042 & $3.160417 E-03$ & 1.84147098 & 1.84540533 & $3.93434 E-03$ \\
\hline
\end{tabular}

investigations have been performed using the powerful artificial neural networks. The comparison of the results is also plotted and overlapping of the proposed and exact solution enhanced more satisfaction of the model. The graphs of absolute error show that most of the values are found in good ranges for all examples of both types, which shows the exactness, worth, and the precision of the designed third-order Emden-Fowler delay differential model.

In the future, the proposed scheme ANN-GA-ASM can be applied as an accurate and efficient stochastic numerical solver for nonlinear singular models [42-44], computational models of fluid dynamics [45-48], fractional models [49-52], and biological models [53-57]. 


\section{Data Availability}

No data were used to support the findings of the study.

\section{Conflicts of Interest}

The authors declare that they have no conflicts of interest.

\section{References}

[1] T. Zhao, "Global periodic-solutions for a differential delay system modeling a microbial population in the chemostat," Journal of Mathematical Analysis and Applications, vol. 193, no. 1, pp. 329-352, 1995.

[2] Y. Kuang, Delay Differential Equations: With Applications in Population Dynamics, Academic Press, Cambridge, MA, USA, 1993.

[3] D. S. Li and M. Z. Liu, "Exact solution properties of a multipantograph delay differential equation," Journal of Harbin Institute of Technology, vol. 32, no. 3, pp. 1-3, 2000.

[4] W. Li, B. Chen, C. Meng et al., "Ultrafast all-optical graphene modulator," Nano Letters, vol. 14, no. 2, pp. 955-959, 2014.

[5] S. I. Niculescu, Delay Effects On Stability: A Robust Control Approach, Vol. 269, Springer Science \& Business Media, Berlin, Germany, 2001.

[6] H. Brunner, Q. Huang, and H. Xie, "Discontinuous Galerkin methods for delay differential equations of pantograph type," SIAM Journal on Numerical Analysis, vol. 48, no. 5, pp. 1944-1967, 2010.

[7] C. H. Hsiao and S. P. Wu, "Numerical solution of timevarying functional differential equations via Haar wavelets," Applied Mathematics and Computation, vol. 188, no. 1, pp. 1049-1058, 2007.

[8] X. T. Wang, "Numerical solution of time-varying systems with a stretch by general Legendre wavelets," Applied Mathematics and Computation, vol. 198, no. 2, pp. 613-620, 2008.

[9] G. Adomian and R. Rach, "A nonlinear differential delay equation," Journal of Mathematical Analysis and Applications, vol. 91, no. 2, pp. 301-304, 1983.

[10] F. Shakeri and M. Dehghan, "Solution of delay differential equations via a homotopy perturbation method," Mathematical and Computer Modelling, vol. 48, no. 3-4, pp. 486498, 2008.

[11] F. Erdogan, M. G. Sakar, and O. Saldır, "A finite difference method on layer-adapted mesh for singularly perturbed delay differential equations," Applied Mathematics and Nonlinear Sciences, vol. 5, no. 1, pp. 425-436, 2020.

[12] A. Shvets and A. Makaseyev, "Deterministic chaos in pendulum systems with delay," Applied Mathematics and Nonlinear Sciences, vol. 4, no. 1, pp. 1-8, 2019.

[13] W. Adel and Z. Sabir, "Solving a new design of nonlinear second-order Lane-Emden pantograph delay differential model via Bernoulli collocation method," The European Physical Journal Plus, vol. 135, no. 6, p. 427, 2020.

[14] R. H. Fowler, "Further studies of emden's and similar differential equations," The Quarterly Journal of Mathematics, vol. 2, no. 1, pp. 259-288, 1931.

[15] Z. Sabir, F. Amin, D. Pohl, and J. L. Guirao, "Intelligence computing approach for solving second order system of Emden-Fowler model," Journal of Intelligent \& Fuzzy Systems, vol. 2020, pp. 1-16, 2020.

[16] Z. Sabir, H. A. Wahab, M. Umar, M. G. Sakar, and M. A. Z. Raja, "Novel design of morlet wavelet neural network for solving second order lane-emden equation," Mathematics and Computers in Simulation, vol. 172, pp. 1-14, 2020.

[17] V. B. Mandelzweig and F. Tabakin, "Quasilinearization approach to nonlinear problems in physics with application to nonlinear ODEs," Computer Physics Communications, vol. 141, no. 2, pp. 268-281, 2001.

[18] V. Radulescu and D. Repovs, "Combined effects in nonlinear problems arising in the study of anisotropic continuous media," Nonlinear Analysis: Theory, Methods and Applications, vol. 75, no. 3, pp. 1524-1530, 2012.

[19] T. Luo, Z. Xin, and H. Zeng, "Nonlinear asymptotic stability of the Lane-Emden solutions for the viscous gaseous star problem with degenerate density dependent viscosities," Communications in Mathematical Physics, vol. 347, no. 3, pp. 657-702, 2016.

[20] M. Ghergu and V. Rădulescu, "On a class of singular GiererMeinhardt systems arising in morphogenesis," Comptes Rendus Mathematique, vol. 344, no. 3, pp. 163-168, 2007.

[21] D. Flockerzi and K. Sundmacher, "On coupled Lane-Emden equations arising in dusty fluid models," Journal of Physics: Conference Series, vol. 268, no. No. 1, Article ID 012006, 2011.

[22] A. Taghavi and S. Pearce, "A solution to the Lane-Emden equation in the theory of stellar structure utilizing the Tau method," Mathematical Methods in the Applied Sciences, vol. 36, no. 10, pp. 1240-1247, 2013.

[23] R. Rach, J.-S. Duan, and A.-M. Wazwaz, "Solving coupled Lane-Emden boundary value problems in catalytic diffusion reactions by the adomian decomposition method," Journal of Mathematical Chemistry, vol. 52, no. 1, pp. 255-267, 2014.

[24] M. Dehghan and F. Shakeri, "Solution of an integro-differential equation arising in oscillating magnetic fields using Hes homotopy perturbation method," Progress in Electromagnetics Research, vol. 78, pp. 361-376, 2008.

[25] K. Boubaker and R. A. Van Gorder, "Application of the BPES to Lane-Emden equations governing polytropic and isothermal gas spheres," New Astronomy, vol. 17, no. 6, pp. 565-569, 2012.

[26] A. H. Bhrawy, A. S. Alofi, and R. A. Van Gorder, "An efficient collocation method for a class of boundary value problems arising in mathematical physics and geometry," Abstract and Applied Analysis, vol. 2014, 2014.

[27] J. I. Ramos, "Linearization methods in classical and quantum mechanics," Computer Physics Communications, vol. 153, no. 2, pp. 199-208, 2003.

[28] J. A. Khan, M. A. Z. Raja, M. M. Rashidi, M. I. Syam, and A. M. Wazwaz, "Nature-inspired computing approach for solving non-linear singular Emden-Fowler problem arising in electromagnetic theory," Connection Science, vol. 27, no. 4, pp. 377-396, 2015.

[29] N. T. Shawagfeh, "Nonperturbative approximate solution for Lane-Emden equation," Journal of Mathematical Physics, vol. 34, no. 9, pp. 4364-4369, 1993.

[30] A.-M. Wazwaz, "A new algorithm for solving differential equations of Lane-Emden type," Applied Mathematics and Computation, vol. 118, no. 2-3, pp. 287-310, 2001.

[31] K. Parand and M. Razzaghi, "Rational Legendre approximation for solving some physical problems on semi-infinite intervals," Physica Scripta, vol. 69, no. 5, p. 353, 2004.

[32] S. Liao, "A new analytic algorithm of Lane-Emden type equations," Applied Mathematics and Computation, vol. 142, no. 1, pp. 1-16, 2003.

[33] C. M. Bender, K. A. Milton, S. S. Pinsky, and L. M. Simmons, "A new perturbative approach to nonlinear problems," 
Journal of Mathematical Physics, vol. 30, no. 7, pp. 1447-1455, 1989.

[34] M. Nouh, "Accelerated power series solution of polytropic and isothermal gas spheres," New Astronomy, vol. 9, no. 6, pp. 467-473, 2004.

[35] Z. Sabir, H. A. Wahab, M. Umar, and F. Erdoğan, "Stochastic numerical approach for solving second order nonlinear singular functional differential equation," Applied Mathematics and Computation, vol. 363, p. 124605, 2019.

[36] M. Umar, F. Amin, H. A. Wahab, and D. Baleanu, "Unsupervised constrained neural network modeling of boundary value corneal model for eye surgery," Applied Soft Computing, vol. 85, Article ID 105826, 2019.

[37] M. Umar, Z. Sabir, and M. A. Z. Raja, "Intelligent computing for numerical treatment of nonlinear prey-predator models," Applied Soft Computing, vol. 80, pp. 506-524, 2019.

[38] M. A. Z. Raja, M. Umar, Z. Sabir, J. A. Khan, and D. Baleanu, "A new stochastic computing paradigm for the dynamics of nonlinear singular heat conduction model of the human head," The European Physical Journal Plus, vol. 133, no. 9, p. 364, 2018.

[39] Z. Sabir, M. A. Manzar, M. A. Z. Raja, M. Sheraz, and A. M. Wazwaz, "Neuro-heuristics for nonlinear singular Thomas-Fermi systems," Applied Soft Computing, vol. 65, pp. 152-169, 2018.

[40] M. A. Z. Raja, J. Mehmood, Z. Sabir, A. K. Nasab, and M. A. Manzar, "Numerical solution of doubly singular nonlinear systems using neural networks-based integrated intelligent computing," Neural Computing and Applications, vol. 31, no. 3, pp. 793-812, 2019.

[41] M. A. Z. Raja, Z. Sabir, N. Mehmood, E. S. Al-Aidarous, and J. A. Khan, "Design of stochastic solvers based on genetic algorithms for solving nonlinear equations," Neural Computing and Applications, vol. 26, no. 1, pp. 1-23, 2015.

[42] Z. Sabir, "On a new model based on third order nonlinear multisingular functional differential equations," Mathematical Problems in Engineering, vol. 2020, 2020.

[43] Z. Sabir, S. Saoud, M. A. Z. Raja, H. A. Wahab, and A. Arbi, "Heuristic computing technique for numerical solutions of nonlinear fourth order Emden-Fowler equation," Mathematics and Computers in Simulation, vol. 2020, 2020.

[44] Z. Sabir, M. A. Z. Raja, J. L. Guirao, and M. Shoaib, "A NeuroSwarming Intelligence Based Computing for Second Order Singular Periodic Nonlinear Boundary Value Problems," Frontiers in Physics, vol. 8, 2020.

[45] M. Umar, Z. Sabir, A. Imran, A. Wahab, M. Shoaib, and M. A. Raja, "Three-dimensional flow of Casson nanofluid over a stretched sheet with chemical reactions, velocity slip, thermal radiation and Brownian motion," Thermal Science, vol. 24, no. 5, pp. 2929-2939, 2019.

[46] Z. Sabir, R. Akhtar, Z. Zhiyu et al., "A computational analysis of two-phase casson nanofluid passing a stretching sheet using chemical reactions and gyrotactic microorganisms," Mathematical Problems in Engineering, vol. 2019, 2019.

[47] T. Sajid, S. Tanveer, Z. Sabir, and J. L. G. Guirao, "Impact of activation energy and temperature-dependent heat source/ sink on maxwell-sutterby fluid," Mathematical Problems in Engineering, vol. 2020, pp. 1-15, 2020.

[48] Y. Jiao and Q. Zheng, "Urea injection and uniformity of ammonia distribution in SCR system of diesel engine," Applied Mathematics and Nonlinear Sciences, vol. 1, 2020.

[49] H. Dehestani, Y. Ordokhani, and M. Razzaghi, "A numerical technique for solving various kinds of fractional partial differential equations via Genocchi hybrid functions," Revista de la Real Academia de Ciencias Exactas, Físicas y Naturales. Serie A. Matemáticas, vol. 113, no. 4, pp. 3297-3321, 2019.

[50] H. Dehestani, Y. Ordokhani, and M. Razzaghi, "On the applicability of Genocchi wavelet method for different kinds of fractional-order differential equations with delay," Numerical Linear Algebra with Applications, vol. 26, no. 5, p. e2259, 2019.

[51] K. Rabiei and Y. Ordokhani, "Solving fractional pantograph delay differential equations via fractional-order boubaker polynomials," Engineering with Computers, vol. 35, no. 4, pp. 1431-1441, 2019.

[52] D. Kaur, P. Agarwal, M. Rakshit, and M. Chand, "Fractional calculus involving (p,q) -mathieu type series," Applied Mathematics and Nonlinear Sciences, vol. 5, no. 2, pp. 15-34, 2020.

[53] Y. G. Sánchez, Z. Sabir, H. Günerhan, and H. M. Baskonus, "Analytical and approximate solutions of a novel nervous stomach mathematical model," Discrete Dynamics in Nature and Society, vol. 2020, pp. 1-9, 2020.

[54] M. Umar, "A stochastic computational intelligent solver for numerical treatment of mosquito dispersal model in a heterogeneous environment," The European Physical Journal Plus, vol. 135, no. 7, pp. 1-23, 2020.

[55] Y. G. Sanchez, "Design of a nonlinear sitr fractal model based on the dynamics of a novel coronavirus (covid)," Fractals, Article ID 2040026, 2020, In press.

[56] F. Evirgen, S. Uçar, and N. Özdemir, "System analysis of HIV infection model with CD4+T under non-singular kernel derivative," Applied Mathematics and Nonlinear Sciences, vol. 5, no. 1, pp. 139-146, 2020.

[57] M. Umar, "Stochastic numerical technique for solving HIV infection model of CD4+ T cells," The European Physical Journal Plus, vol. 135, no. 6, p. 403, 2020. 